\title{
Analysis and Modelling of Willingness to Receive Reward for Relay in Ad Hoc Networks
}

\author{
Naoyuki Karasawa ${ }^{1,2}$, Kyoko Yamori ${ }^{32}$, Kenji Donkai ${ }^{2}$, \\ and Yoshiaki Tanaka ${ }^{2,4}$ \\ 1 Department of Information Engineering, Niigata University \\ 2-8050 Ikarashi, Nishi-ku, Niigata, 950-2181 Japan \\ karasawa@ie.niigata-u.ac.jp \\ 2 Global Information and Telecommunication Institute \\ Waseda University \\ 1-3-10 Nishi-Waseda, Shinjuku-ku, Tokyo, 169-0051 Japan \\ 3 Department of Business Administration, Asahi University \\ 1851 Hozumi, Mizuho-shi, Gifu, 501-0296, Japan \\ 4 Research Institute for Science and Engineering, Waseda University \\ 17 Kikuicho, Shinjuku-ku, Tokyo, 162-0044 Japan \\ kyamori@alice.asahi-u.ac.jp, ken-don@toki.waseda.jp, ytanaka@waseda.jp
}

\begin{abstract}
In ad hoc networks, relay nodes use their limited resources such as battery capacity, CPU, buffer, etc. to support other nodes' communications This discourages users from joining an ad hoc network and becoming a relay node. Receiving a reward for supporting relay may encourage users to join such networks. This research focuses on the factors of residual battery power, and time during which $\mathrm{AC}$ power supply is not available. In this paper, the willingness to receive reward for relay in ad hoc networks is investigated by questionnaire survey. The relation between such factors and the willingness to receive rewards for relay is analysed quantitatively and models of willingness to receive reward for relay are estimated.
\end{abstract}

\section{Introduction}

An ad hoc network consists of self-organizing nodes using multi-hop relay. In ad hoc networks, a source node communicates with a destination node via a multi-hop path using other nodes as relay nodes when the source and the destination nodes are out of communication range of each other. While relaying, relay nodes use their limited resources such as battery capacity, CPU, buffer, etc. to support other nodes'

Please use the following format when citing this chapter:

Karasawa, N., Yamort, K., Donkai, K., Tanaka, Y, 2007, in IFIP International Federation for Information Ptocessing, Volume 252, Integration and Innovation Orient to F-Socicty Volume 2, eds. Wang, W., (Boston: Springer), pp. $160-167$. 
communications. This discourages users from joining ad hoc networks and becoming a relay node.

Charging / rewarding methods [1], [2] have been proposed as methods for overcoming problems that occur with relay. In ad hoc networks that use charging / rewarding methods, all nodes have some initial currency. Source nodes can communicate with destination nodes via a path consisting of relay nodes only if the source node can pay at least one unit of currency to each relay node. Nakano et al. [3] showed that charging / rewarding methods reduce unfairness in ad hoc networks and decrease the variance among the nodes induced by frequency of relay. Therefore, rewarding relay nodes can offset disadvantages for relay such as consumption of battery power and processing loads, etc.

In this paper, we investigate the willingness to receive reward for relay using a questionnaire survey in an ad hoc network where relay nodes receive rewards for relay. We focus on residual battery power and the time during which AC power supply is not available. The relation between such factors and the willingness to receive reward for relay is analysed quantitatively. We also discuss how such factors influence willingness to receive reward for relay. Finally, some models of willingness to receive reward for relay are estimated.

\section{Questionnaire Survey on the Willingness to Receive Reward for Relay}

An overview of the questionnaire survey used to investigate the willingness to receive reward (WTR) for relay in ad hoc networks is described. In this paper, WTR for relay is defined as a reward that is capable of motivating users to relay other nodes' communications. Suppose that ad hoc networks use charging / rewarding methods, which is illustrated in Fig.1. In such ad hoc networks, the relay node receives a reward for relay in proportion to the amount of communication when it relays other nodes' communications.

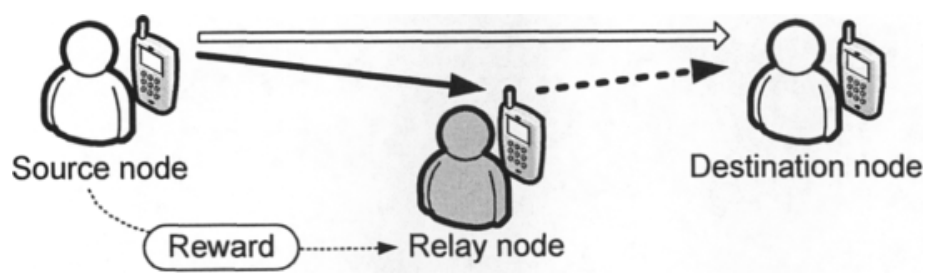

Fig. 1. Ad hoc network using charging / rewarding method.

WTR for relay seems to be affected by several factors. This paper focuses on a type of mobile terminal, the residual battery power during relay, and the time during which AC power supply is not available. In particular, it is expected that WTR for relay depends on residual battery power and the time during which $\mathrm{AC}$ power supply 
is not available. The questionnaire survey is designed to test the following hypothesis.

Hypothesis: There are positive correlations between battery power consumption and WTR for relay and between AC power unavailability duration and WTR for relay.

Here, battery power consumption means the total amount of battery power consumed by several functions of a mobile terminal. $\mathrm{AC}$ power unavailability duration means the time during which $\mathrm{AC}$ power supply is not available. To test the hypothesis, it is necessary to quantify the relations between various factors and WTR for relay.

Suppose that each node in an ad hoc network uses a mobile terminal. For example, a cellular phone with a wireless LAN device or a laptop PC with a wireless LAN is considered as mobile terminal. In this questionnaire survey, power consumption for relay is not considered, as it is negligible in comparison with the power consumption by LCD display, CPU, and so on. The node is able to use a mobile terminal for 6 hours when the battery power is at 1, i.e. fully charged. The questionnaire assumes various battery conditions. Namely, the battery power consumption is $0,0.5,0.75$, and 0.92 , and the $\mathrm{AC}$ power unavailability duration is 3 , 6 , and 12 hours. The response format of the questionnaire requires the respondent to input an arbitrary value as their WTR for relay with a duration of one minute.

\section{Analysis of Questionnaire Results}

There are 194 effective responses to the questionnaire (male 131, female 63). The WTR of each sample is normalized so that the maximum value is 1 . Only if all WTR values are 0 , the normalized WTR values are set to 0 . The normalized WTR is summed for each condition and their mean values are taken to be the mean opinion scores (MOS). The MOS thus obtained is used to estimate a WTR curve using regression analysis. Linear, logarithmic, exponential, and power approximations are used for approximation. We determine whether each of the four functions is applicable from their contribution coefficients.

The WTR curve for the relation between WTR and battery power consumption is best approximated as shown in (1),

$$
U=\alpha_{r} \exp \left(\beta_{r} r\right)
$$

where battery power consumption is represented as $r$ and WTR as $U$. The values of parameters $\alpha_{r}$ and $\beta_{r}$ and the contribution coefficient $R^{2}$ are shown in Table 1.

Table 1. Parameters $\alpha_{r}$ and $\beta_{r}$ in (1).

\begin{tabular}{llll}
\hline AC ower unavailability duration [hours] & $\alpha_{r}$ & $\beta_{r}$ & $R^{2}$ \\
\hline 3 & 0.287 & 0.715 & 0.889 \\
6 & 0.344 & 0.748 & 0.925 \\
12 & 0.462 & 0.725 & 0.912 \\
\hline
\end{tabular}


For the relation between WTR and the AC power unavailability duration, the WTR curves estimated by linear approximation are as shown in (2),

$$
U=\alpha_{t} t+\beta_{t},
$$

where the AC power unavailability duration is represented by $t$ and the WTR by $U$. Parameters $\alpha_{t}$ and $\beta_{t}$ and contribution coefficients $R^{2}$ are shown in Table 2 .

Table 2. Parameters $\alpha_{t}$ and $\beta_{t}$ in (2).

\begin{tabular}{llll}
\hline Battery power consumption & $\alpha_{t}$ & $\beta_{t}$ & $R^{2}$ \\
\hline 0 & 0.0205 & 0.241 & 0.999 \\
0.5 & 0.0254 & 0.297 & 0.998 \\
0.75 & 0.0330 & 0.367 & 0.997 \\
0.92 & 0.0411 & 0.498 & 1.00 \\
\hline
\end{tabular}

Consequently, the WTR curves of the battery power consumption and the WTR curves of the $\mathrm{AC}$ power unavailability durations are significant because the contribution coefficients of the estimated regression formulae are sufficiently large. Figs. 2 and 3 show the WTR curves of the battery power consumption and the WTR curves of the $\mathrm{AC}$ power unavailability duration, respectively.

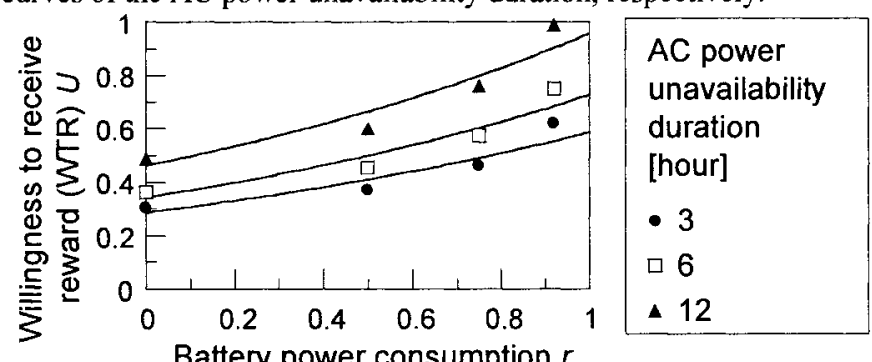

Fig.2. WTR $U$ and battery power consumption $r$.



Fig. 3. WTR $U$ and AC power unavailability duration $t$.

To establish a method of estimating WTR for any battery condition in all cases, we analyse and discuss the relation of factors in the WTR for relay. In this paper, it is 
assumed that the WTR for relay depends on the battery conditions of the mobile terminal. Battery power consumption and $\mathrm{AC}$ power unavailability duration are considered as battery conditions. Therefore, the WTR curve is defined by,

$$
U=f\left(U_{r}, U_{t}\right)
$$

Here, $U_{r}$ is a variable dependent on battery power consumption and $U_{t}$ is a variable dependent on $\mathrm{AC}$ power unavailability duration.

There are two assumptions in (3). It is assumed that $U_{r}$ and $U_{1}$ are factors that influence $U$ independently and that $U_{r}$ and $U_{t}$ are correlated factors that influence $U$. In this paper, using these assumptions, we devise WTR curves that fit each model.

\section{Estimating the WTR Curve: Independent Factors}

In this section, it is assumed that $U_{r}$ and $U_{t}$ are independent of each other. Then, the relation between $U_{r}$ and $U_{t}$ is given in the form of their sum and we obtain (4).

$$
U=a_{0}+a_{1} U_{r}+a_{2} U_{t},
$$

where $a_{0}, a_{1}$, and $a_{2}$ are parameters. $U_{r}$ can be approximated by $k_{r} \alpha, \exp \left(\beta_{r} r\right)$ where $k_{r}$ is constant because the relation between WTR and battery power consumption can be approximated by exponential curves from (1). $U_{t}$ can be approximated by $k_{t}\left(\alpha_{t} t+\right.$ $\beta_{t}$ ) where $k_{t}$ is constant because the relation between WTR and the AC power unavailability duration can be approximated by linear equations from (2). So, (4) is denoted by (5).

$$
U=a_{0}^{\prime}+a_{1}^{\prime} \exp \left(\beta_{r} r\right)+a_{2}^{\prime} t,
$$

where $a_{0}^{\prime}, a_{1}^{\prime}$ and $a_{2}^{\prime}$ are parameters. Consider $\exp \left(\beta_{r} r\right)$ in (5). In general, it is known that $\exp (x)$ is represented by

$$
\exp (x)=\sum_{n=0}^{\infty} \frac{1}{n !} x^{n}
$$

In this paper, (7) is assumed because the numerical results of $\exp \left(\beta_{r} r\right)$ are sufficiently close to the numerical results of (6) when $x=\beta_{r} r$ and $n=2$.

$$
\exp \left(\beta_{r} r\right) \approx 1+\frac{1}{1 !}\left(\beta_{r} r\right)+\frac{1}{2 !}\left(\beta_{r} r\right)^{2}=1+\beta_{r} r+\frac{\beta_{r}^{2}}{2} r^{2} .
$$

Using (7) in (5), (8) is obtained.

$$
U=a_{0}^{n}+a_{1}^{\prime \prime} r+a_{2}^{\prime \prime} r^{2}+a_{3}^{\prime \prime} t,
$$

where $a_{0}^{\prime \prime}, a_{1}^{\prime \prime}, a_{2}^{\prime \prime}$, and $a_{3}^{\prime \prime}$ are parameters.

To estimate the WTR curve with the best fit, the WTR curve estimated by (5) and the WTR curve estimated by (8) are compared. First, to devise WTR curve with (5), multiple regression analysis is used. In this multiple regression analysis, $\exp \left(\beta_{r} r\right)$ 
and $t$ are denoted by predictor variables, and $U$ is denoted by criterion variable. As a result, WTR curve is shown as follows:

$$
U=-0.260+0.400 \exp \left(\beta_{r} r\right)+0.0299 t,
$$

where $\beta_{r}$ are determined by $t$. The contribution coefficient of (9) is 0.918 .

Consider $\beta_{r}$ in (9). As mentioned in Table $1, \beta_{r}$ is obtained by constrained time $t$. Thus the curve fitting $\beta_{r}$ using various approximation methods are studied. However, we cannot select curves with a good fit because their contribution coefficients are not sufficiently large. Table 1 shows that $\beta_{r}$ is irregularly distributed within a narrow range. So, it is assumed that $\beta_{r}$ is determined by the mean values. From Table 1, the mean value of $\beta_{r}$ is 0.730 . We substitute this value into (9).

$$
U=-0.260+0.400 \exp (0.730 r)+0.0299 t .
$$

The contribution coefficient of $(10)$ is 0.917 .

Then, let us devise WTR curve by (8). For (8), multiple regression analysis is used. In this multiple regression analysis, $r, r^{2}$, and $t$ are denoted by the predictor variables, and $U$ is denoted by the criterion variable. The WTR obtained by multiple regression analysis curve is shown by (11).

$$
U=0.177-0.177 r+0.651 r^{2}+0.0300 t .
$$

The contribution coefficient of (11) is 0.970 .

Deliberate the WTR curve estimated by (5) and the WTR curve estimated by (8). The contribution coefficient of each WTR curve is sufficiently large. Hence, each WTR curve is significant. In the derivation from (5), $\beta_{r}$ is approximated by mean values as an approximate equation. However, there is a model by which the contribution coefficient of an approximate equation of $\beta_{r}$ is not sufficiently large. It is considered that the contribution coefficient affects the predictive accuracy of the WTR curve in this model. By contrast, (8) is estimated by approximation using (6). Using (6), it is possible to show (8) basically in the form of a polynomial equation even if degree of a polynomial equation increases.

\section{Estimating the WTR Curve: Correlated Factors}

In this section, it is assumed that $U_{r}$ and $U_{t}$ are correlated. Under this assumption, the relational expression between $U_{r}$ and $U_{r}$ is given in the form of the product and (12) is obtained.

$$
U=b_{0} U_{r} U_{,},
$$

where $b_{0}$ is parameter. As in the previous section, substituting $k_{r} \alpha_{r} \exp \left(\beta_{r} r\right)$ and $k_{t}\left(\alpha_{t} t\right.$ $+\beta_{t}$ ) into $U_{r}$ and $U_{t},(12)$ is described in (13).

$$
U=b_{0}\left\{a_{r} \exp \left(\beta_{r} r\right)\right\}\left(\alpha_{t} t+\beta_{t}\right) .
$$

Then, taking a logarithm of both sides of (13), (13) is denoted as follows: 


$$
\log (U)=b_{0}^{\prime}+b_{1}^{\prime} r+b_{2}^{\prime} \log \left\{1+\left(\alpha_{t} / \beta_{t}\right)\right\},
$$

where $b_{0}^{\prime}, b_{1}^{\prime}$ and $b_{2}^{\prime}$ are parameters. Consider $\log \left\{1+\left(\alpha_{t} / \beta_{t}\right) t\right\}$. In general, it is known that $x$ is greater than $\log (1+x)$ when $x$ is greater than 0 . For simplicity, (15) is assumed by using this relation.

$$
\log \left\{1+\left(\alpha_{t} / \beta_{t}\right) t\right\} \approx\left(\alpha_{t} / \beta_{t}\right) t
$$

Using (15) in (14), (16) is obtained as follows:

$$
\log (U)=b_{0}^{\prime \prime}+b_{1}^{\prime \prime} r+b_{2}^{\prime \prime} t
$$

where $b_{0}{ }_{0}, b_{1}$ and $b{ }_{2}$ are parameters.

To estimate WTR curve that have good fit, WTR curve estimated by (14) and WTR curve estimated by (16) are compared. First, in order to devise WTR curve by (14), multiple regression analysis is used. In this multiple regression analysis, $r$ and $\log \left\{t+\left(\beta_{t} / \alpha_{t}\right)\right\}$ are denoted by the predictor variables, and $\log (U)$ is denoted by the criterion variable. The WTR curve resulting from the multiple regression analysis is shown as follows (See Appendix):

$$
U=0.0182 \exp (0.732 r)(t+11.7) \text {. }
$$

The contribution coefficient of (17) is 0.937 .

Let us devise WTR curves using (16) and multiple regression analysis in which $r$ and $t$ are denoted by the predictor variables, and $\log (U)$ is denoted by the criterion variable. The resulting WTR curve is shown as follows:

$$
U=0.247 \exp (0.729 r) \exp (0.0527 t) \text {. }
$$

The contribution coefficient of (18) is 0.934 .

Deliberate the WTR curve estimated by (14) and the WTR curve estimated by (16). The contribution coefficient of each WTR curve is sufficiently large. Hence, each WTR curve is significant. In particular, it is considered that (18) is simple because there are fewer variables in the WTR curve estimated by (16) than in the WTR curve estimated by (14). For each parameter of (12), the coefficient that affects the whole type is equivalent to $b_{0}$ of (12). Then, $b_{0}$ of (12) means users' underlying parameters for WTR for relay.

\section{Conclusion}

In this paper, we focused on the residual battery power and the time during which AC power supply is not available as factors in WTR for relay in ad hoc networks as investigated by questionnaire survey. Based on the results of the questionnaire survey, the relation between battery-related factors and WTR for relay is analysed quantitatively. Two models are considered in this paper: one in which the factors are independent and the other in which the factors are correlated. The best-fit curves of the WTR by relay are estimated in both models. The contribution coefficients of the WTR curves estimated by using both assumptions are sufficiently large. 
Consequently, both assumptions produce models with good fits for the WTR for relay.

In future work we intend to analyse WTR for relay along with other factors.

\section{References}

1. Buttyan, L., Hubaux, J.: Stimulating cooperation in self-organizing mobile ad hoc networks: ACM/Kluwer Mobile Networks and Applications (MONET) (2003) 579-592.

2. Salem, N.B., Buttyan, L., Hubaux, J.P., Jakobsson, M.: A charging and rewarding scheme for packet forwarding in multi-hop cellular networks: 4th ACM Symposium on Mobile Ad Hoc Networking and Computing (MobiHoc 2003) (2003) 13-24.

3. Nakano, K., Panta, R., Sengoku, M., Shinoda, S.: On performance of a charging/rewarding scheme in mobile ad-hoc networks: 2005 IEEE International Symposium on Circuits and Systems (ISCAS 2005), vol.3. (2005) 2962-2966.

\section{Appendix: Derivation of (17)}

The derivation of (17) is described. From (14),

$$
\log (U)=b_{0}^{m}+b_{1}^{m} r+b_{2}^{m} \log \left(t+\beta_{i} / \alpha_{\imath}\right)
$$

where $b^{\prime \prime \prime}, b^{\prime \prime \prime}{ }_{1}$ and $b^{\prime \prime \prime}{ }_{2}$ are parameters. Then, multiple regression analysis is used. In this multiple regression analysis, $r$ and $\log \left(t+\beta_{t} / \alpha_{t}\right)$ are denoted by the predictor variables, and $\log (U)$ is denoted by the criterion variable. The WTR curve resulting from the multiple regression analysis is shown as follows:

$$
\log (U)=-4.00+0.732 r+1.02 \log \left(t+\beta_{t} / \alpha_{t}\right),
$$

where $\alpha_{t}$ and $\beta_{t}$ are determined by $r$. The contribution coefficient of (A2) is 0.957 .

From Table 2, $\alpha_{t}$ and $\beta_{t}$ are obtained by $r$ and regression analysis is used to estimate $\alpha_{t}$ and $\beta_{t}$. As a result, $\alpha_{t}$ and $\beta_{t}$ are approximated by exponential approximation. Then, the estimated results are shown in (A3) and (A4).

$$
\begin{aligned}
\alpha_{t} & =0.0195 \exp (0.731 r), \\
\beta_{t} & =0.228 \exp (0.729 r) .
\end{aligned}
$$

The contribution coefficients of (A3) and (A4) are 0.933 and 0.894 , respectively. Calculating $\beta_{t} / \alpha_{t}$ using (A3) and (A4), $\beta_{t} / \alpha_{t}$ is $11.7 \exp (-0.002 r)$. From this result, we obtain (A5).

$$
\log (U)=-4.00+0.732 r+1.02 \log \{t+11.7 \exp (-0.002 r)\} .
$$

Consider $1.02\{t+11.7 \exp (-0.002 r)\}$ in (A5). In this paper, to simplify a WTR curve, (A5) is approximated by (A6). Therefore, (17) is obtained from (A6).

$$
\log (U)=-4.00+0.732 r+\log (t+11.7) \text {. }
$$

